\title{
High and low states of the system AM Herculis
}

\author{
K. $\mathrm{Wu}^{1,2}$ and L. L. Kiss ${ }^{2}$ \\ 1 Mullard Space Science Laboratory, University College London, Holmbury St Mary, Surrey RH5 6NT, UK \\ e-mail: kw@mssl.ucl.ac.uk \\ 2 School of Physics A28, University of Sydney, NSW 2006, Australia \\ e-mail: 1.kiss@physics.usyd.edu.au
}

Received 27 August 2007 / Accepted 24 October 2007

\section{ABSTRACT}

\begin{abstract}
Context. We investigate the distribution of optically high and low states of the system AM Herculis (AM Her). Aims. We determine the state duty cycles, and their relationships with the mass transfer process and binary orbital evolution of the system.

Methods. We make use of the photographic plate archive of the Harvard College Observatory between 1890 and 1953 and visual observations collected by the American Association of Variable Star Observers between 1978 and 2005. We determine the statistical probability of the two states, their distribution and recurrence behaviors.

Results. We find that the fractional high state duty cycle of the system AM Her is 63\%. The data show no preference of timescales on which high or low states occur. However, there appears to be a pattern of long and short duty cycle alternation, suggesting that the state transitions retain memories. We assess models for the high/low states for polars (AM Her type systems). We propose that the white-dwarf magnetic field plays a key role in regulating the mass transfer rate and hence the high/low brightness states, due to variations in the magnetic-field configuration in the system.
\end{abstract}

Key words. accretion, accretion disks - stars: binaries: close - stars: individual: AM Herculis - stars: magnetic fields stars: variables: general - stars: novae, cataclysmic variables

\section{Introduction}

Magnetic cataclysmic variables $(\mathrm{mCVs})$ are close interacting binaries containing a Roche-lobe filling low-mass companion star, usually an $\mathrm{M}$ dwarf, transferring material to a magnetic white dwarf. Most mCVs have orbital periods around $1.3-5 \mathrm{~h}$, and the magnetic fields of the white dwarfs in mCVs are sufficiently strong $\left(B \sim 10^{6}-10^{8} \mathrm{G}\right)$ that the accretion flow is fieldchannelled near the white dwarf. mCVs are strong X-ray emitters, and many $\mathrm{mCV}$ s were first identified in X-ray surveys.

The two main subclasses of mCVs are the polars and the intermediate polars. Polars are also known as AM Herculis type systems. Unlike other cataclysmic variables and binary $\mathrm{X}$-ray sources, polars do not have an accretion disk. All components in the system are locked by a strong white-dwarf magnetic field into synchronized (or almost synchronized) rotation. Intermediate polars contains white dwarfs with weaker magnetic fields. An intermediate polar has an accretion disk, but the inner disk is truncated by the white-dwarf magnetic field. White dwarfs in intermediate polars are often fast-spinning, with a spin rate roughly 10 times higher than the rate of the orbital rotation. For a review of cataclysmic variables and polars, see Cropper (1990); Warner (1995); Wu (2000); Beuermann (2002); Wu et al. (2003).

Polars are found to show alternating bright and faint optical/X-ray states, commonly referred to as high and low states, respectively. In the absence of an accretion disk, the high and low states of polars are likely to be consequences of changes in the mass-transfer rate in the system. It has been argued that the high/low states of polars are related to the atmospheric magnetic activity of the mass-donor star. In the star-spot model (Livio \& Pringle 1994), a low state is caused by a temporary cessation of mass transfer, due to a magnetic spot on surface of the Rochelobe filling mass-donor star traversing the inner Lagrange (L1) point. While we may have some understanding of the long-term variations in the mass transfer of cataclysmic variables, we are still uncertain what causes the high and low states and the state transitions of polars.

The bright polar AM Herculis $(\mathrm{AM}$ Her $=4 \mathrm{U} 1813+50$, with a visual magnitude $V \sim 13-15.5$ and an orbital period of $185.6 \mathrm{~min}$ ) has been monitored in the optical bands for more than 100 years. The optical brightness of the source has shown large variations. An early study (Feigelson et al. 1978) claimed that there was no strong evidence of a well defined low state. A later study (Götz 1993), however, showed the presence of high and low states with good statistical significance. On the other hand, analysis of the fraction of polars in high and low states during the XMM-Newton and ROSAT X-ray survey observations (Ramsay et al. 2004) suggested that polars spend roughly half of their time in a high state and half in a low state. While surveys of ensembles of systems might establish the effective duration of the high/low state duty cycles, to determine the characteristics of the state transitions and the driving mechanisms we need to analyze the long-term behavior of individual systems.

In this paper we present a quantitative analysis of high/low states and their transition for the polar AM Her. We use the photometric data of the system taken in the last 115 years. We assess the models for the state transitions and propose a possible scenario for the process. We organize the paper as follows. In Sect. 2 we present the data and analysis; in Sect. 3 we comment on the 


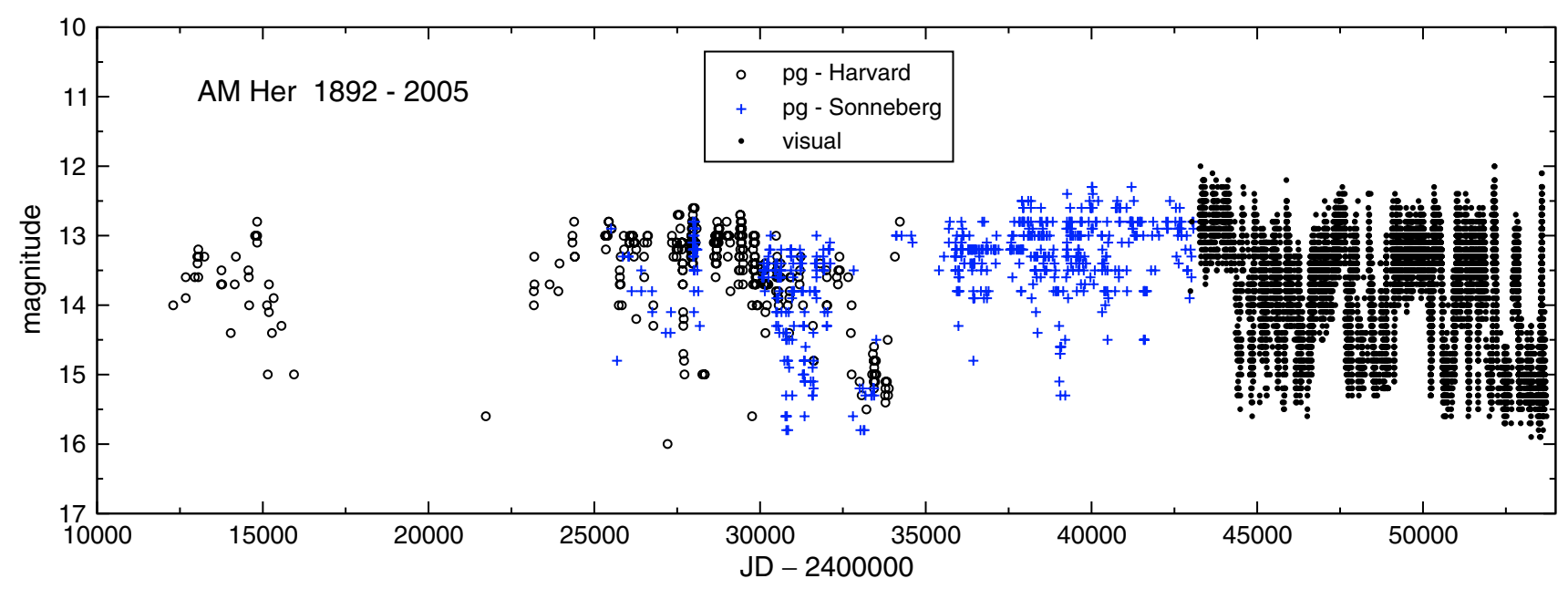

Fig. 1. The combined photographic and visual light curve of the system AM Her between 1892 and 2005 (negative detections omitted).

statistics and in Sect. 4 we discuss the magnetic spot model and an alternative. A brief summary is given in Sect. 5 .

\section{Data sources and analysis}

Our study is based on three different datasets, which allowed the reconstruction of AM Her's light curve for most of the last 115 years: (i) photographic magnitudes determined by the second author on 348 blue plates from the Harvard College Observatory's plate collection; (ii) 600 photographic magnitudes measured by Hudec \& Meinunger (1977) on Sonneberg plates; (iii) 17704 visual observations collected by the American Association of Variable Star Observers (AAVSO). The combined light curve is plotted in Fig. 1, which shows that the last $\sim 85$ years are covered with almost no long (i.e. extending many years) gaps.

The earliest observations are those of the Harvard patrol program. The historic light curve from the Harvard plates was already obtained by Feigelson et al. (1978); however, those data were never published in tabulated form. Moreover, we found 378 useful blue plates in the archive, which is 40 more than the sample analyzed by Feigelson et al. (1978). Amongst these, we could detect AM Her on 348 plates. Photographic magnitudes were then estimated with reference to a sequence of 10 nearby comparison stars uniformly covering the brightness range from $B=12.6$ to $B=16.3$. The magnitude values were taken from The Guide Star Catalog (GSC 2.2, STScI 2001). The brightness estimates of AM Her were made visually through a $9 \times$ viewing eyepiece with an estimated photometric accuracy of about $\pm 0.1-0.2 \mathrm{mag}$. The earliest plate was taken on August 1, 1890, while the first positive detection was on July 18, 1892; the last detection was recorded by us on July 23, 1952. We ignored the few yellow plates because their temporal coverage was very poor. Also, a few blue plates from 1976-1977 were omitted from the analysis because for that period the other two sets provided a full coverage.

It is worth noting that contrary to what Feigelson et al. (1978) found, our Harvard magnitudes are in agreement with those of Hudec \& Meinunger (1977). In 23 cases when the Harvard and Sonneberg plates were taken on the same day, the mean Sonneberg minus Harvard magnitude was $0.08 \pm 0.27 \mathrm{mag}$; since AM Her can show much larger variations over a time-scale of hours, the small mean difference provides reassurance about the homogeneity of the two photographic datasets.
The third and the most continuous dataset comes from the hundreds of visual observers of the AAVSO. These observations were begun in mid-1977 and have been continuous ever since. The first 20 years of the data were analyzed by Hessman et al. (2000) in terms of mass-transfer variations in AM Her. The present set is 7.5 years longer, containing almost 18000 individual observations. Since we are interested in the transitions between high and low states, we calculated 10-day bins. This way, we averaged out rapid brightness fluctuations that occur on shorter time-scales and appear as an additional "noise" in the light curve.

The photographic data are less useful for determining the statistics of the high and low states. Compared to the visual observations, the photographic ones are quite sparse. The mean distance between two consecutive Harvard plates is 35 days (for the more continuous coverage between JD 2423000 and 2433000 ), while the Sonneberg plates were taken only slightly more frequently (one point in every 30 days). For comparison, the mean distance between two consecutive 10-day bins is 10.9 days, which shows that the duty cycle of the visual data was about $90 \%$ with the 10-day sampling (and still over 70\% with 3-day sampling). Nevertheless, the photographic curve can still be used to check the range of brightness fluctuations in the high state over a time scale of a century. Figure 1 reveals that the overall brightness distribution did not change significantly in the last 113 years. There are hints for gradual change in the maximum brightness, similarly to the trend seen in the visual data, but the full $\sim 3$ mag peak-to-peak amplitude was quite stable. It is also remarkable how similar the photographic and visual magnitudes were. From the mean values of the maximum and minimum brightnesses we could not infer any measurable zero-point shift (greater than 0.1-0.2 mag).

Figure 2 shows the 10-day binned visual light curve, which is dominated by the transitions between high and low states. To measure the time-spans of these, we defined a boundary between them at $m_{\text {vis }}=14.0$ mag (dashed line in Fig. 2). This way, we could include the short-lived (10-30 days) and fainter (13.5-14.0 mag) maxima among the high states. We then measured the durations of high and low states as the time-spans of being brighter or fainter than $14.0 \mathrm{mag}$. Since the typical fading or rising times ranged from 10 to 30 days, this simplification has only slightly affected the measured lengths, ranging from 10 to 1400 days and 10 to 700 days for the high and low states, respectively. 


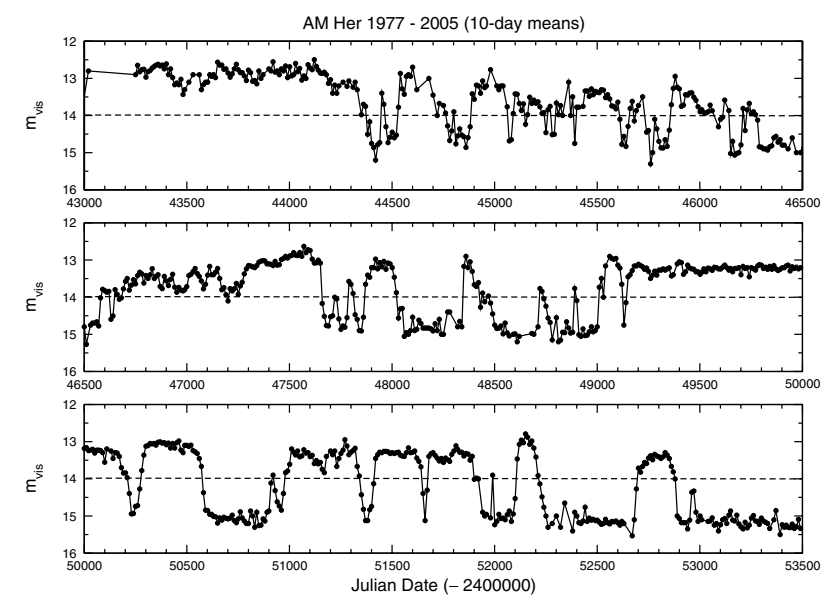

Fig. 2. The binned AAVSO light curve. The dashed lines show the adopted boundary between high and low state at $m_{\mathrm{vis}}=14.0 \mathrm{mag}$.

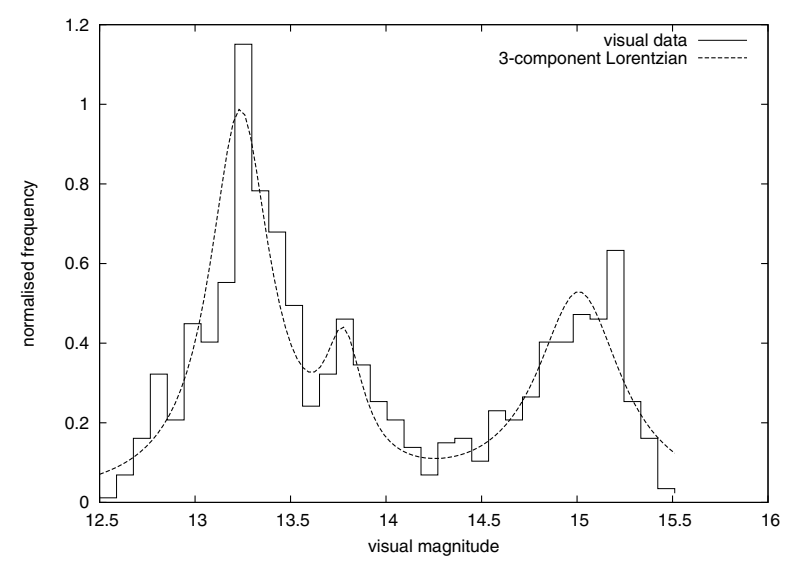

Fig. 3. The histogram of the binned AAVSO light curve with a threecomponent Lorentzian fit.

\section{Results}

\subsection{Distribution of high and low states and duration of duty cycles}

The normalized histogram of the binned visual light curve (Fig. 3) clearly shows the two main states of the star. Furthermore, there is a smaller third peak centered at $13.7 \mathrm{mag}$, which corresponds to the longer periods of fainter maxima in the top panel of Fig. 2 and the short/faint high states in the middle and bottom panels of Fig. 2. The integral under the curve from 12.5 to 14.0 mag gives a measure of the total duration of high states, which is $62 \%$, when adding up the area under the steps of the histogram.

To improve the duration calculation, we tried to find a good analytic fit to the histogram. The best results were given by two- and three-component Gaussian and Lorentzian functions. Of these, the three-component Lorentzian profile yielded the smallest residual mean scatter. In Table 1 we give the best fit parameters of the four functions and corresponding rms values. The Lorentzian fits give $\sim 20 \%$ smaller rms, which reflects the observation that the peaks in the histogram are rather sharp. The integral of the analytic fit gives $62.8 \%$ as the total duration of the high state, which we adopt as the best estimate of this parameter with less than $1 \%$ uncertainty (but, of course, subject to systematic bias arising from the definiton of the high state).
Table 1. Best fit Gaussian $\left(f(x)=C_{1} \mathrm{e}^{-(x-\mu)^{2} / 2 \sigma^{2}}\right)$ and Lorentzian $\left(f(x)=C_{2}\left[1+((x-\mu) / \Gamma)^{2}\right]^{-1}\right)$ functions to the histogram of the binned visual light curve.

\begin{tabular}{lrrr}
\hline \hline Function & Parameters & & rms \\
\hline 2 Gaussians & & & 0.140 \\
& $\mu_{1}$ & $13.29 \pm 0.04$ & \\
& $\sigma_{1}$ & $0.36 \pm 0.04$ & \\
& $\mu_{2}$ & $14.97 \pm 0.05$ & \\
& $\sigma_{2}$ & $0.29 \pm 0.05$ & \\
\hline 3 Gaussians & & & 0.122 \\
& $\mu_{1}$ & $13.23 \pm 0.03$ & \\
& $\sigma_{1}$ & $0.23 \pm 0.03$ & \\
& $\mu_{2}$ & $14.97 \pm 0.04$ & \\
& $\sigma_{2}$ & $0.28 \pm 0.04$ & \\
& $\mu_{3}$ & $13.84 \pm 0.07$ & \\
& $\sigma_{3}$ & $0.17 \pm 0.06$ & \\
\hline 2 Lorentzians & & & 0.116 \\
& $\mu_{1}$ & $13.25 \pm 0.02$ & \\
& $\Gamma_{1}$ & $0.26 \pm 0.03$ & \\
& $\mu_{2}$ & $15.01 \pm 0.04$ & \\
& $\Gamma_{2}$ & $0.28 \pm 0.07$ & \\
\hline 3 Lorentzians & & & 0.101 \\
& $\mu_{1}$ & $13.23 \pm 0.02$ & \\
& $\Gamma_{1}$ & $0.19 \pm 0.03$ & \\
& $\mu_{2}$ & $15.01 \pm 0.03$ & \\
& $\Gamma_{2}$ & $0.27 \pm 0.05$ & \\
& $\mu_{3}$ & $13.78 \pm 0.04$ & \\
& $\Gamma_{3}$ & $0.12 \pm 0.07$ & \\
\hline
\end{tabular}

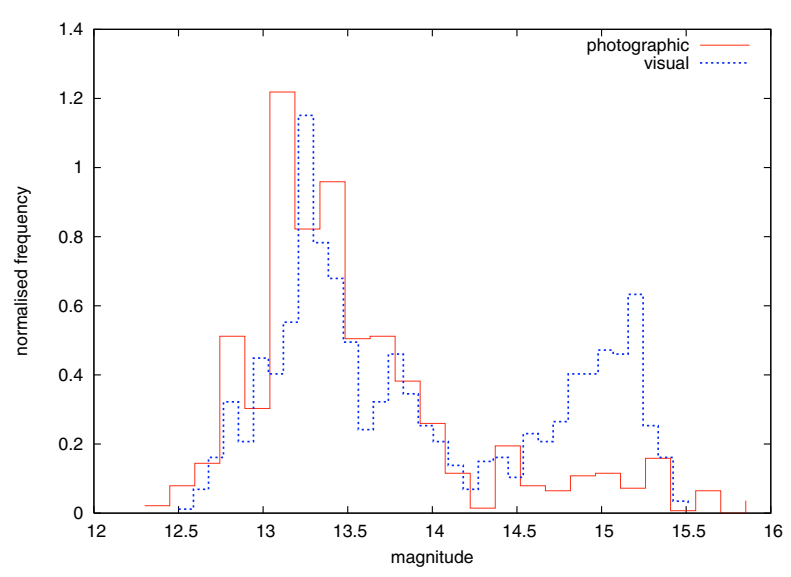

Fig. 4. A comparison of the histograms of the photographic and visual data. Note the lack of faint photographic observations.

We also calculated the histogram of the photographic light curve. For this, we combined the Harvard and Sonneberg magnitudes, as there is no systematic difference between them. In Fig. 4 we compare it to the histogram of the visual light curve. Note that the magnitude bins in the histograms are slightly different, in order to avoid oversampling of the sparser photographic curve. The close agreement of the brighter peaks indicates the stability of the full magnitude range of the high state over the last century. The secondary peak at $\sim 13.7$ mag is not resolved, but the broader shoulder of the main peak suggests the presence of that distinct feature in the early data. The low state, on the other hand, is heavily affected by the magnitude limits of the patrol plates. Nevertheless, the extension of the fainter peak is very similar in both histograms, so that it is very likely that the statistics of the low state also stayed very similar throughout the 20th century. 


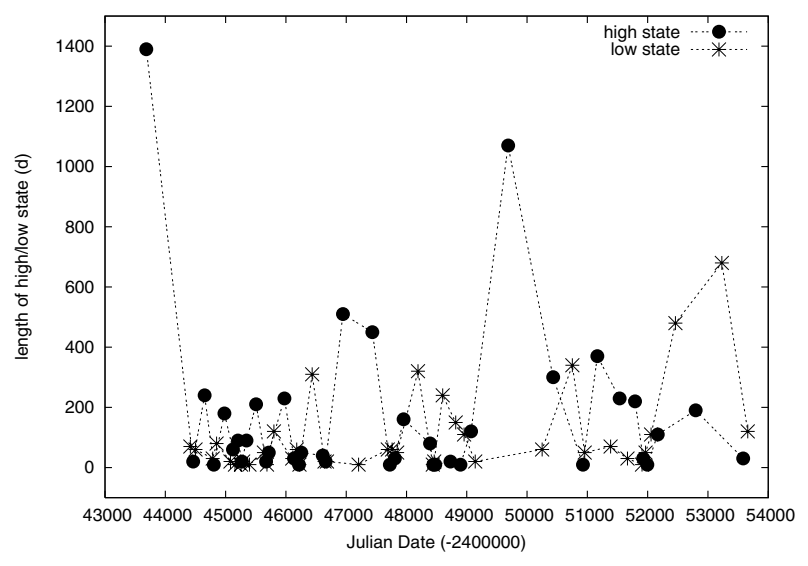

Fig. 5. Time variations of the duration of high/low states.

We find a remarkable behavior in the duration of the high and low states. In Fig. 5 we plot the time-spans of the two states as a function of time, where the durations are assigned to the midtimes of each state. There seems to be a well-defined recurrence in both states, in the sense that alternating long and short states occur over time. If this pattern is not due to statistical coincidence, the system has retained certain memory of the previous states and the transitions.

\subsection{Comparison with results from $X$-ray surveys}

In the ROSAT X-ray survey of 28 polars, 16 were in the low state, and in the XMM-Newton X-ray survey of the 37 systems, 21 were in the low state. Bayesian analyses give a probability of 0.585 for the polars in the low state, with the $90 \%$ confident intervals being $(0.342,0.863)$, for the ROSAT sample, and a probability of 0.442 , with the $90 \%$ confident interval being $(0.258,0.734)$, for the XMM-Newton sample (Ramsay et al. 2004). Moreover, there were no significant differences in the global distributions of orbital periods among the high-state and the low-state systems in either the ROSAT or XMM-Newton samples. The high-state and the low-state systems were not statistically biased toward long or short orbital periods, and there was no evidence of period clumping of high- and low-state systems. The distributions of the high-state and the low-state systems were consistent with random orders, when ranked according to their orbital periods. The findings that half of the sources are in the high state and the other half in the low state, and that the high and low states of the systems are unrelated to the system orbital periods suggest a 50/50 high-low state duty cycle for individual systems.

Our analysis of the AAVSO optical photometric data showed that the system AM Her has spent about $60 \%$ of its time in the high state. If polars generally have the same high-low state duty cycle as the system AM Her, we would expect that $3 / 5$ of the systems will be in their high state in a snap-shot survey. The inference of the X-ray survey observations of Ramsay et al. (2004) that the high/low-state fractional duty cycle of an individual polar is about $50 \%$ is therefore consistent with the observed highlow state duty cycles of the system AM Her in the last 100 years.

\section{Models for high/low states}

\subsection{Variations in optical brightness and mass transfer}

The total bolometric luminosity of a polar is the sum of its hard X-ray, soft X-ray, UV and optical luminosities, i.e.
$L_{\mathrm{bol}}=L_{\mathrm{hx}}+L_{\mathrm{sx}}+L_{\mathrm{uv}}+L_{\mathrm{opt}}$. The hard X-rays are free-free emission from the shock-heated accretion gas at the bottom region of the accretion column. The soft X-rays probably originate from the thermalized atmosphere of the accretion pole bombarded by dense accretion blobs. The UV radiation is generally interpreted as reprocessed emission of the X-rays, and its luminosity is roughly proportional to the X-ray luminosity. In the high state, the optical emission is dominated by the cyclotron emission from the shock-heated gas near the whitedwarf surface. In the low state, where cyclotron emission from the accretion gas is negligible, the optical luminosity is mainly due to the emission of the low-mass mass-donor star. From the BeppoSAX, ROSAT and EXOSAT observations, Hessman et al. (2000) demonstrated a scaling relation $L_{\mathrm{opt}} \propto L_{\mathrm{bol}}^{\gamma}$ for the system AM Her in a bright (high) state, where mass transfer occurred. The index parameter $\gamma \approx 2$ for $V$ magnitude $<15$.

The accretion luminosity of an accreting white dwarf is $L_{\mathrm{acc}}=-G M_{\mathrm{wd}} \dot{M} / R_{\mathrm{wd}}$, where $G$ is the gravitational constant, $M_{\mathrm{wd}}$ and $R_{\mathrm{wd}}$ are the mass and radius of the white dwarf, respectively, and $\dot{M}$ is the mass transfer rate. For polars in a high state, $L_{\text {acc }}$ is much higher than the intrinsic luminosity of the two stars. Thus, we have $L_{\mathrm{bol}} \approx L_{\mathrm{acc}}$. Polars are Roche-lobe filling systems, with the mass transfer rate given by

$\dot{M} \approx-A_{\mathrm{L} 1} c_{\mathrm{s}} \rho_{0} \exp \left[-(\Delta r / H)^{2}\right]$

(Lubow \& Shu 1975; Papaloizou \& Bath 1975; Meyer \& Meyer-Hofmeister 1983), where $c_{\mathrm{s}}$ is the gas sound speed at the L1 point, $\rho_{0}$ is the characteristic atmospheric density of the companion star, $H$ is the atmospheric scale height of the companion star, and $\Delta r$ is the difference between the effective Roche-lobe volume radius and the radius of the companion star. The effective area of the nozzle of the gas stream at the first Lagrangian (L1) point, $A_{\mathrm{L} 1}$, is given by

$A_{\mathrm{L} 1}=\frac{2 \pi c_{\mathrm{s}} a^{3}}{G M_{\mathrm{wd}}(1+q) k(q)}$,

where $a$ is the orbital separation, $q$ is the ratio of the mass of the secondary star to the white dwarf, and $k(q)$ is a function with value $\approx 7$ (see Hessman et al. 2000; Meyer \& Meyer-Hofmeister 1983).

The visual magnitude $V$ of an astrophysical object is related to the optical luminosity: $V=-\log _{10} L_{\text {opt }}+\lambda$, where $\lambda$ is a constant determined by the distance, and the bolometric and photometric corrections. It follows that for a polar we have

$V=-\gamma\left[\log _{10} \rho_{0}+2 \log _{10} c_{\mathrm{s}}-\left(\log _{10} e\right) \xi^{2}\right]+\lambda^{\prime}$,

where $\xi=\Delta r / H$. When the system parameters $\lambda, M_{\mathrm{wd}}, a$ and $q$ are specified, the quantity $\lambda^{\prime}$ is a "constant". The visual magnitude $V$, however, is an observable quantity (which is a random variable) determined by the indirect observables $\rho_{0}$, $c_{\mathrm{s}}$, and $\Delta r / H$ (which are also random variables). Any variations/fluctuations in these indirectly observable variables would lead to variations/fluctuations in the visual magnitude $V$. It follows from Eq. (3) that

$(\delta V)^{2}=\left(\frac{\gamma}{\ln 10}\right)^{2}\left[\left(\frac{\delta \rho_{0}}{\rho_{0}}\right)^{2}+\left(\frac{2 \delta c_{\mathrm{s}}}{c_{\mathrm{s}}}\right)^{2}\right]+\left(\gamma \log _{10} e\right)^{2}(2 \xi \delta \xi)^{2}$.

The two-Gaussian and two-Lorentzian fits to the optical brightness variations of the AAVSO data of the system AM Her both yield a mean brightness $\mu_{1} \approx 13.3$, and a standard deviation $\sigma_{1} \approx 0.3$ (see Sect. 2 ) for the high state. This gives a characteristic high-state brightness variation $\delta V \sim \sigma_{1} \approx 0.3$. In order to 
explain the spread in the optical brightness during the high state, fluctuations of either $\left(\delta \rho_{0} / \rho_{0}\right) \sim 0.4,\left(\delta c_{\mathrm{s}} / c_{\mathrm{s}}\right) \sim 0.2$ or $\delta \xi \sim 0.2$ (for $\xi \sim 1$ ) are required (Eq. (4)). The low-state mean brightness is about 15.0 and the standard deviation is about 0.05 . The difference between the optical brightness of the high and low states is $\delta \mu=\mu_{2}-\mu_{1} \approx 1.7$. During the low state the optical luminosity is contributed mainly by the emission from the mass-donor star and the heated white dwarf (see Bonnet-Bidaud et al. 2000). Therefore we can use the brightness variation to constrain the variations of $\rho_{0}, c_{\mathrm{s}}$ or $\xi$.

\subsection{Star-spot model and magnetic-locking model}

The magnetic-spot model (Livio \& Pringle 1994) attributes the occurrence of the low state to the cessation or reduction of the mass-transfer rate when a magnetic-star spot on the surface of the mass-donor secondary star traverses the inner Lagrangian point. If the area covered by the star spot is not a large fraction (say less than 50\%) of the total stellar surface area, the high state is a "default" state, where the mass transfer is uninterupted. Unless there is an underlying mechanism to synchronize the movement of magnetic spots on the stellar surface, the migration of the star spot to the inner Lagrangian point is random, and the occurrence of low state and the duration of the low state can be modelled by Poisson processes. Moreover, one would also expect that brightness (mass-transfer) variations during the high state are Poisson/Gaussian-type fluctuations, provided that the distribution of the hot-spot size is not narrow and resembling a $\delta$-function.

Analysis by Hessman et al. (2000) had highlighted some difficulties of the generic star-spot model. In addition, the star-spot model has difficulties in explaining why this kind of high-low state phenomenon occurs in all polars but does not occur in other cataclysmic variables (magnetic or non-magnetic) and low-mass $\mathrm{X}$-ray binaries in general, when all these systems have late-type low-mass secondary stars similar to those in polars.

From the long-term brightness variations of the system AM Her, we have quantified three properties of its high state. Firstly, there is a spread of brightness $(\delta V \approx 0.3)$ during the high state. The value of the spread is robust - it is insensitive to whether Gaussian fits or Lorentzian fits are used; it is also insensitive to whether a two-component fit or a three-component fit is used. Secondly, Lorentzian fits to the $V$-magnitude variations give better statistics than Gaussian fits, in the two-component model as well as the three-component model. Thirdly, there is an additional weaker high state (at $V \approx 13.8$, for Lorenztian fits) in addition to the usual high and low states (at $V \approx 13.2$ and 15.1 respectively, for Lorentzian fits). Moreover, the occurrence and duration of high and low states are not well described as Poission processes, and the alternation of the two states appears to show certain memories (see Fig. 5). Given these properties and the uniqueness of the high/low state in polars, we argue that the high/low states of polars are unlikely to be simply caused by random migration of magnetic star spots of the secondary star to the inner Lagrangian point.

In a low-mass close binary system, the magnetic field of the compact star generally has negligible direct effects on the system orbital dynamics. However, in a polar, the magnetic field of the compact primary star is so strong that it affects the accretion flow and the orbital dynamics of the system. This makes polars very unique among all low-mass close binary systems. We propose that the high/low states and their transitions are caused by variations in the magnetic interaction between the white dwarf and the mass-donor secondary star. In other words, the magnetic field of the white dwarf can alter the mass transfer rate. In certain contexts the situation resembles that in the RS CVn systems, where the magneto-activities of a component star are greatly influenced by the magnetic field of its companion star (see e.g. Uchida \& Sakurai 1985). This is in contrast with the star-spot model, where the magnetic field of the mass-donor plays the central role.

In the canonical model of polars (Chanmugam \& Wagner 1977), the magnetic stress exerted by the white-dwarf field is strong enough to disrupt the formation of an accretion disk in the white-dwarf Roche lobe. Moreover, all components in the binary are magnetically locked into synchronous rotation with one single period (Wu \& Wickramasinghe 1993; see also Joss et al. 1979; Hameury et al. 1987; Campbell 1990).

The orbital separation of a polar is given by

$a=4.8 \times 10^{9}\left[\left(\frac{M_{\mathrm{wd}}(1+q)}{M_{\odot}}\right)\left(\frac{P_{\mathrm{o}}}{3 \mathrm{hr}}\right)^{2}\right]^{1 / 3} \mathrm{~cm}$,

where $P_{\mathrm{o}}$ is the orbital period. The Roche-lobe volume radius of the white dwarf is given by

$R_{\mathrm{L}}=a\left(0.500+0.227 \log _{10} q\right)^{-1}$

(Plavec \& Kratochvil 1964). The surface polar field of the white dwarf in system AM Her is about 20 MG (Schmidt et al. 1981; Bonnet-Bidaud et al. 2000). The mass-donor of the system AM Her is a M 4.5-M 5 star (Latham et al. 1981). For a white-dwarf of $\approx 0.75 M_{\odot}, R_{\mathrm{L}} \sim 2.9 \times 10^{9} \mathrm{~cm}$. If the whitedwarf magnetic field is dipolar, the field strength would be about $140 \mathrm{kG}$ at the inner Lagrangian point. The observed mean surface magnetic field strengths of late-type $M$ stars are $\sim 5 \mathrm{kG}$ (Johns-Krull \& Valenti 1996), and theoretical models predicted field strengths around $10 \mathrm{kG}$ (see Buzasi 1997). These values are significantly smaller than the field exerted by the magnetic white dwarf. The white-dwarf magnetic field plays a more dominant role in determining the magnetospheric activity in the inner Lagrangian point, where mass transfer from the donor star initiates.

The surface temperature of the mass-donor M star of the system AM Her is about $3250 \mathrm{~K}$ (Bailey et al. 1991). In the absence of external forces, the photospheric pressure is estimated to be $\sim 10^{3}-10^{4} \mathrm{erg} \mathrm{cm}^{-3}$ (see e.g. Edwards \& Pringle 1987). The energy density of the stellar field of the mass-donor star is $\sim 10^{6} \mathrm{erg} \mathrm{cm}^{-3}$. At the inner Lagrangian point, the energy density of the white-dwarf magnetic field is, however, greater than $10^{9} \mathrm{erg} \mathrm{cm}^{-3}$. The atmospheric scale height $H$ is therefore determined by an external force, due to the white-dwarf magnetic field.

If the brightness variations in the high state of the system AM Her are caused by mass transfer variations, it would require $10-20 \%$ variations either in the sound speed $c_{\mathrm{s}}$ at the L1 point, the atmosphere base density $\rho_{0}$, or the quantity $\Delta r / H$ (see Sect. 4.1). Although one cannot dogmatically reject the possibility of large amplitude variations in the surface temperature and base density of the mass-donor star, providing a sensible explanation for the cause of such atmospheric variations is not easy. The more likely causes would be the variations in $\Delta r / H$. The quantity $\Delta r$ is the difference between the stellar radius and the Roche-lobe volume radius. It varies with the secular evolutionary timescale of the binary orbit, which is much longer than the timescales of the brightness fluctuations of the system. Variations in the local magnetic field strength or geometrical configuration may result in a change in the atmospheric scale height $H$ of the mass-donor star. The local magnetic field at the 
L1 point is jointly determined by the white-dwarf magnetic moment and its orientation, the stellar atmospheric magnetic field, and the dynamics of the mass transfer flow. The linear width of the nozzle at the $\mathrm{L} 1$ point is $\approx \sqrt{A_{\mathrm{L} 1}} \sim 10^{9} \mathrm{~cm}$ (Eq. (2)), and the local sound speed is $\sim 10^{6} \mathrm{~cm} \mathrm{~s}^{-1}$ (inferred from the atmospheric temperature of $3250 \mathrm{~K}$ ). It follows that the shortest timescale allowed for the brightness variations is $\sim 10^{3} \mathrm{~s}$, which is the sound crossing time. This is the minimum timescale on which the geometry of mass transfer flow is readjusted in response to changes in the magnetic-field configuration at the vicinity of the L1 point. Note that as the mass transfer rate has an exponential dependence on $(\Delta r / H)^{2}$ (Eq. (1)), a reduction of $H$ by a factor of 3 would be be enough to quench the mass transfer.

Although the component stars in polars are expected to be magnetically locked into synchronous rotation, perfect locking is an idealisation rather than a reality. Several polars have been found to show asynchronous white-dwarf spin and orbital rotation (e.g. BY Cam, Silber et al. 1992; Honeycutt \& Katfla 2005). Spin-orbit synchronism is preserved only if the torque generated by the accretion flow is counter-balanced by the magnetic torque. There are observations that the locations of the accretion spots in certain polars changed in different epochs. An interpretation is that spin-orbital asynchronism is continually perturbed, as the accretion flow and hence the accretion torque are not steady (Bailey et al. 1993; Wu et al. 1994).

The high-low state and the transition fit very well in this scenario. If the magnetic field configuration is sufficiently perturbed, the mass transfer will be suppressed, and the new equilibrium configuration is established for the zero accretion torque situation. This also provides a consistent explanation for an intermediate state (Fig. 3), which is, in contrast, not easily explained in the frame-work of the conventional star-spot model. Moreover, our analysis of the brightness distribution showed Lorentzians rather than Gaussians, especially for the high and the intermediate states, suggesting that the spread of the brightness might not be due to purely random fluctuations but controlled by certain underlying resonant processes. A possible explanation for such resonant is that the locking occurs at the minimum energy field configuration in the presence of a multipolar white-dwarf magnetic field (Wickramasinghe \& Wu 1991; Wu \& Wickramasinghe 1993), and any small perturbations will lead to oscillations of the relative orientation of the magnetic field and hence the mass transfer rates and the brightness of the system. When the oscillations are dampened, it naturally gives rise to Lorentzian type profiles for the brightness distribution.

There are two necessary conditions for the operation of the high-low state-transition mechanism described above. Firstly, the system must be magnetically locked or quasi-magnetically locked. Secondly, the magnetic field of the compact star must overwhelm the magnetic field of the mass-donor star at the L1 point. These conditions are satisfied in polars but not in the weaker field $\mathrm{mCVs}$ and other binary systems. Here, we elaborate this briefly in the case of intermediate polars. Consider an intermediate polar with the same orbital parameters and stellar masses as the system AM Her, but a white-dwarf surface polar field strength of $\sim 1 \mathrm{MG}$ (see Wickramasinghe et al. 1991). This field is not strong enough to disrupt the entire accretion disk and to enforce spin-orbit synchronism (see Chanmugam \& Wagner 1977). Without screening, the white-dwarf magnetic field is about $7 \mathrm{kG}$ at the L1 point for a dipolar field configuration, and it is weaker if higher-order multi-pole field components are present. An accretion disk is a body of fast differentially rotating conducting fluid. Its presence causes field screening. The actual strength of the white-dwarf magnetic field at the L1 point is significantly lower than $7 \mathrm{kG}$, and the corresponding energy density is significantly smaller than $10^{6} \mathrm{erg} \mathrm{cm}^{-3}$. The magnetic field of the mass-donor M dwarf, however, reaches $\sim 5-10 \mathrm{kG}$, giving an energy density of $\sim 10^{6} \mathrm{erg} \mathrm{cm}^{-3}$. The stellar field is therefore strong enough to suppress the magnetic harassment at the L1 point by the white dwarf. This allows the M star to selfadjust to establish an atmospheric scale height that facilitates the mass transfer process. Note that polars could have evolved from long-period intermediate polars, but long-period polars would not evolve to become shorter-period intermediate polars (Chanmugam \& Ray 1984; King et al. 1985; Wickramasinghe et al. 1991). Observations have shown a deficiency of intermediate polars with $P_{\mathrm{o}}<2 \mathrm{hr}$ in comparison with polars. Dipolar magnetic field scales with $r^{-3}$ and high order multi-pole fields have stronger $r$ dependences (where $r$ is the radial distance from the white dwarf). Thus, the white-dwarf magnetic field at the L1 point scales with $P_{\mathrm{o}}^{-\alpha}$ roughly, where $\alpha \geq 2$. The influence of the white-dwarf magnetic field on the mass-donor $\mathrm{M}$ star is generally insignificant for intermediate polars. Therefore, intermediate polars are not expected to show high/low states and state transitions like those observed in polars.

In summary, while the star-spot model has highlighted the importance of magnetism in the role for the high and low states and the state transition of polars, analyses have shown that the model has fallen short of satisfactorily explaining the 100-year data of optical brightness variations of the system AM Her (see Hessman et al. 2000). Here, we propose an alternative magnetic model. The key regulator of the mass transfer is the global magnetic field of the white dwarf, instead of the local magnetic field variations at the surface of the mass-donor star. The states correspond to various equilibrium locking configurations in the presence or absence of the accretion torque. The Lorentzian distribution of brightness variation can be attributed by dampened oscillations around the equilibrium configuration. This scenario is the same frame-work of synchronism of polars as a unique class of interacting binaries. It also reconciles with the observations that prominent high/low states occur in all polars, in which the white-dwarf magnetic field is strong enough to affect the dynamics of the whole system, but not in intermediate polars or non-magnetic cataclysmic variables, in which the white-dwarf magnetic field is too weak to influence accretion flow at the distance of the mass-donor star, or to alter the orbital dynamics of the system.

\section{Conclusion}

We investigate the high/low states of the system AM Her using three sources of archival optical data: the photographic plates of the Harvard College Observatory between 1890 and 1953, the visual observations by the American Association of Variable Star Observers between 1978 and 2005, and the published photographic data from Sonneberg plates. We determine the statistical probability of the high and low states, their distribution and recurrence behaviors. The distribution of the high states is well fitted by a Lorentzian. There appears to be an intermediate state. The fractional high state duty cycle of the system AM Her is $63 \%$, which is consistent with the inference that the duty cycle of the high state of polars is roughly $50 \%$ based on the ROSAT and $X M M$-Newton survey observations. The data show no preference of timescales on which high or low states occur. There appears a pattern of long and short duty cycle alternation, suggesting that the state transitions retain certain memories. We propose that the high/low states are caused by variations in the magnetic field configuration of the system, and that the magnetic field of the 
white dwarf plays the key role in regulating the mass flow at the inner Lagrangian point. This scenario may overcome certain difficulties unexplained by the star-spot model, where the cessation of mass transfer is caused by magnetic star spots of the mass-donor star traversing the Lagrangian point.

Acknowledgements. K.W.'s visit to the University of Sydney was supported by the NSW State Expatriate Researcher Award. L.L.K. has been supported by a University of Sydney Postdoctoral Research Fellowship. We sincerely thank variable star observers of the AAVSO whose dedicated observations over three decades made this study possible. L.L.K. thanks the kind hospitality of Dr. Arne Henden, the Director of the AAVSO and all the staff members at the AAVSO Headquarters (Cambridge, MA) during his visit in early 2006. We are also grateful to Dr. Charles Alcock, the Director of the Harvard College Observatory, for access to the Photographic Plate Collection, and to Alison Doane, the Curator of the Collection, for helpful assistance.

\section{References}

Bailey, J., Ferrario, L., \& Wickramasinghe, D. T. 1991, MNRAS, 251, 37P Bailey, J., Wickramasinghe, D. T., Ferrario, L., Hough, J., \& Cropper, M. 1991, MNRAS, 251, 37P

Beuermann, K. 2002, PASP, 114, 427

Bonnet-Bidaud, J. M., et al. 2000, A\&A, 354, 1003

Buzasi, D. L. 1997, ApJ, 484, 855

Campbell, C. G. 1990, MNRAS, 244, 367

Chanmugam, G., \& Ray, A. 1984, ApJ, 285, 252

Chanmugam, G., \& Wagner, R. L. 1977, ApJ, 213, L13

Cropper, M. 1990, Sp. Sci. Rev., 54, 195

Edwards, D. A., \& Pringle, J. E. 1987, MNRAS, 229, 383
Feigelson, E., Dexter, L., \& Liller, W. 1978, ApJ, 188, 55

Götz, W. 1993, Astron. Nachr., 314, 69

Hameury, J. M., King, A. R., \& Lasota, J.-P. 1987, A\&A, 171, 140

Hessman, F. V., Gänsicke, B. T., \& Mattei, J. A. 2000, A\&A, 361, 952

Honeycutt, R. K., \& Katfla, S. 2005, MNRAS, 364, 97

Hudec, R., \& Meinunger, L. 1977, Mitt. Veränderl. Sterne, 8, 10

Johns-Krull, C. M., \& Valenti, J. A. 1996, ApJ, 459, L95

Joss, P. C., Katz, J. I., \& Rappaport, S. A. 1979, ApJ, 230, 176

King, A. R., Frank, J., \& Ritter, H. 1985, MNRAS, 213, 181

Latham, D. W., Liebert, J., \& Steiner, J. E. 1981, ApJ, 246, 919

Livio, M., \& Pringle, J. E. 1994, ApJ, 427, 956

Lubow, S. H., \& Shu, F. H. 1975, ApJ, 198, 383

Meyer, F., \& Meyer-Hofmeister, E. 1983, A\&A, 121, 29

Papaloizou, J. C. B., \& Bath, G. T. 1975, MNRAS, 172, 339

Plavec, M., \& Kratochvil, P. 1964, Bull. Astr. Czech., 15, 165

Ramsay, G., Cropper, M., Wu, K., et al. 2004, MNRAS, 350, 1373

Schmidt, G. D., Stockman, H. S., \& Margon, B. 1981, ApJ, 243, L159

Silber, A., Brandt, H. V., Ishida, M., Ohashi, T., \& Remillard, R. A. 1992, ApJ, 389, 704

Space Telescope Science Institute 2001, The Guide Star Catalog, Version 2.2, VizieR On-line Data Catalog: I/271, STScI, Baltimore

Uchida, Y., \& Sakurai, T. 1985, IAUS, 107, 281

Warner, B. 1995, Cataclysmic Variable Stars (Cambridge: Cambridge University Press)

Wickramasinghe, D. T., \& Wu, K. 1991, MNRAS, 253, 11P

Wickramasinghe, D. T., Wu, K., \& Ferrario, L. 1991, MNRAS, 249, 460

Wu, K. 2000, Sp. Sci. Rev., 93, 611

Wu, K., \& Wickramasinghe, D. T. 1993, MNRAS, 260, 141

Wu, K., Wickramasinghe, D. T., Bailey, J., \& Tennant, A. F. 1994, PASA, 11, 198

Wu, K., Cropper, M., Ramsay, G., Saxton, C. J., \& Bridge, C. 2003, Chin. J. Astron. Astrophys., 3 (Suppl.), 235 